\title{
Optionen für den Einstieg in den Arbeitsmarkt oder: Ein Lehrstück für einen gescheiterten Politikwechsel ${ }^{*}$
}

\author{
Von Rolf G. Heinze** und Wolfgang Streeck***
}

Zusammenfassung: Der Beitrag thematisiert zunächst die Rahmenbedingungen auf dem Arbeitsmarkt und die spezifische Krise des deutschen Beschäftigungssystems (stabile Koinzidenz hoher Arbeitslosigkeit mit einer niedrigen Erwerbsquote). Ziel der Arbeitsmarkt- und Beschäftigungspolitik müsste es sein, eine auch in anderen westeuropäischen Ländern zu beobachtende Beschäftigungsdynamik im Dienstleistungssektor zu entfalten. Eine wesentliche Barriere gegen die Dienstleistungsbeschäftigung (vor allem bei einfachen, gering produktiven Tätigkeiten) ist die "Kostenkrankheit", so dass eine Politik der Beschäftigungsexpansion die relativen Preise gerade von beschäftigungsintensiven Diensten reduzieren muss. Im Beitrag werden zwei Optionen näher diskutiert: einerseits die Senkung der Bruttolöhne von Arbeitnehmern, die nicht favorisiert wird, und andererseits die Senkung der Sozialversicherungsbeiträge. Eine solche selektive Senkung der Beiträge auf niedrige Einkommen wird auch von anderen politischen Institutionen und Wissenschaftlern empfohlen. Dennoch sind (u. a. im „Bündnis für Arbeit") erhebliche Widerstände entstanden, so dass ein institutioneller Wechsel der Politik noch nicht stattgefunden hat.

Summary: This article deals with the general conditions on the labor market and the specific employment crises in Germany (stabile coincidence of high unemployment with a low labor force participation rate). Therefore, the goal of labor market policies should be to develop more dynamic employment in the service sector as can be observed in other Western European countries as well. An essential barrier to service sector activities (above all in simple, low productive operations) is "cost sickness", necessitating that the measures of labor market policy reduce relative prices, especially in the case of laborintensive jobs. Two options are discussed in detail: on the one hand the reduction of the gross salary of employees, which is not favored, and on the other hand the reduction of social insurance contributions. A selective reduction of the contributions by low-income workers is also recommended by other political institutions and experts, yet in Germany considerable resistance has emerged (e.g. in the "alliance for work") and, as a result, political changes have not yet taken place.

\section{$1 \quad$ Rahmenbedingungen auf dem Arbeitsmarkt}

Auch im Sommer des Jahres 2002 (nach fast vier Jahren rot-grüner Bundesregierung) hat sich die Situation auf dem Arbeitsmarkt strukturell kaum verändert. Wir haben weiterhin ein massives Beschäftigungsdefizit und ein institutionell fest verankertes Repertoire gescheiterter Methoden zu seiner Bekämpfung. Der angekündigten Senkung der Arbeitslosenzahlen auf 3,5 Mill. zum Trotz waren im Juli und August wiederum über 4 Mill. arbeitslos gemeldet; rund 1 Mill. Personen verweilen in Weiterbildungs- und anderen arbeitsmarktpoliti-

\footnotetext{
* Manuskript abgeschlossen im September 2002

* * Ruhr-Universität Bochum, Fakultät für Sozialwissenschaft, E-Mail: Rolf.Heinze @ ruhr-uni-bochum.de

* * * Max-Planck-Institut für Gesellschaftsforschung, Köln, E-Mail: streeck@ mpi-fg-koeln.mpg.de
} 
schen Maßnahmen. Bereits ohne die „stille Reserve“ (die für 2001 auf rd. 1,9 Mill. Personen geschätzt wird) sind damit in Deutschland über 5 Mill. Arbeitsuchende zu registrieren, wobei nicht alle gesellschaftlichen Gruppen gleichermaßen betroffen und auch massive regionale Differenzen unübersehbar sind. Gleichzeitig mehren sich die Anzeichen gravierender Mängel in der Qualifikation.

Die strukturell verfestigte Massenarbeitslosigkeit lähmt die Kräfte, die zur Modernisierung von Wirtschaft und Gesellschaft nötig gebraucht werden. Der Vorsitzende der im Sommer 2002 in den Medien breit rezipierten Kommission „Moderne Dienstleistungen am Arbeitsmarkt“" Peter Hartz, hat Recht, wenn er in dem Abschlussbericht schreibt: „Arbeitslosigkeit raubt den Menschen die Fähigkeiten und ist damit Verschwendung von schöpferischem Potential. Der Schaden trifft die Arbeitslosen und unser Land. Arbeitslosigkeit fördert die Spaltung der Gesellschaft. Über deren Gräben verstehen sich die Menschen nicht mehr, sie sprechen eine andere Sprache, Zeitperspektiven verschieben sich. Denk- und Verhaltensweisen haben nur noch wenig miteinander zu tun. Die Angst, durch Arbeitsplatzverlust aus dem gesellschaftlichen Ganzen vertrieben zu werden, durchdringt alle Lebenszusammenhänge.“

So richtig es sein mag, nachdrücklich auf die Dimension des sozialen Problems hinzuweisen, auch um die notwendige Unterstützungsbereitschaft der verantwortlichen politischen und gesellschaftlichen Akteure zu gewinnen, so wenig originell ist die Botschaft. Schon seit der Marienthalstudie aus den 30er Jahren von Marie Jahoda (1975) und anderen ist klar, welch fundamentale Bedeutung der Arbeitsplatz für die soziale Anerkennung und die Selbstachtung hat, zumal nach fast vier Jahren einer rot-grünen Regierungskoalition sich schon die Frage stellt, warum sich die Blockaden auf dem deutschen Arbeitsmarkt nicht gelöst haben und welche Kräfte für die Politik der Nichtentscheidungen bzw. Klientelentscheidungen verantwortlich sind. Jüngst hat der ehemalige Bundeskanzler Helmut Schmidt auf diesen neuen Reformstau und auf die Akteure hingewiesen, die weiterhin an dem deutschen Pfad der Sozial- und Arbeitsmarktpolitik mit hohen Sozialbeiträgen und Sozialausgaben festhalten und damit eine Lösung der Beschäftigungskrise behindern, aber in der Problemdiagnose der Politiker explizit nicht benannt werden: „Vielen Politikern fehlt es auf diesem Felde an Mut zur Wahrheit. Sie fürchten die Agitation des Machtkartells aus Gewerkschaften und Arbeitgeberverbänden und die negative Reaktion der Betroffenen. Den beiden größten Gewerkschaften sollte deutlich gesagt werden: Ihr vertretet im Ergebnis eurer Politik zwar die kurzfristigen Interessen der aktiven Arbeitnehmer, aber zur Überwindung der Arbeitslosigkeit trägt eure Politik nichts bei. Der Arbeitgeberorganisation sollte vorgehalten werden: Ihr vertretet zwar die kurzsichtigen Interessen eurer Unternehmen, aber die finanziellen Folgen eurer Politik schiebt ihr auf den Staat ab, das heißt: auf den Steuerzahler" (ders. 2002: 10, 2002: 10). Die Aufwendungen für diese klassisch defensive und einvernehmlich von Regierung, Opposition und den Tarifverbänden verfolgte Arbeitsmarktpolitik sind enorm und haben längst die Grenzen der öffentlichen Belastbarkeit erreicht: „Jährlich werden über 70 Mrd. Euro für aktive Arbeitsmarktpolitik, Lohnersatzleistungen und die Sicherung des Lebensunterhalts für arbeitslose Menschen aufgewandt (Bundesanstalt für Arbeit: 54 Mrd. Euro; Bund für Arbeitslosenhilfe: 13 Mrd. Euro; Kommunen für Sozialhilfe: 5 Mrd. Euro)“(Kommission 2002: 37).

Die hohe Subventionierung der seit Jahrzehnten bestehenden Massenarbeitslosigkeit hat starke Steuer- und Beitragsbelastungen zur Folge, die ihrerseits die Dynamik auf dem bundesdeutschen Arbeitsmarkt einschnüren. Die Sozialleistungsquote hat sich mit rund 34\% 
des Bruttosozialprodukts in den letzten Jahren nicht verändert und liegt im internationalen Vergleich ähnlich hoch wie am Ende der christlich-liberalen Regierungskoalition unter Bundeskanzler Kohl 1998. Die rot-grüne Bundesregierung hat auch ihr anderes Versprechen, die Absenkung des gesamten Sozialbeitragssatzes auf unter $40 \%$, nicht einlösen können.

Die eigentliche Krise des deutschen Beschäftigungssystems besteht in einer langfristig stabilen Koinzidenz hoher Arbeitslosigkeit mit einer niedrigen Erwerbsquote. Nur mit einer Erhöhung der Beschäftigtenrate (etwa auf skandinavisches Niveau) ließe sich die mit der Arbeitsmarktkrise zusammenhängende Krise der sozialen Sicherungssysteme nachhaltig lindern. Die deutschen Beschäftigungsquoten (d.h. der Anteil der Beschäftigten an der Bevölkerung im erwerbsfähigen Alter von 15 bis 65 Jahren) unterscheiden sich auch nach Beschäftigungsgruppen deutlich von denen in anderen Ländern. Während die Beschäftigungsquote der Frauen in Deutschland bei 57,8\% liegt, beträgt sie in den Niederlanden 63,4\%, in Großbritannien 64,5\%, in Schweden 69,7\% und in Dänemark sogar 72,1\%. Man braucht keine gewagten und politisch umstrittenen Vergleiche mit dem amerikanischen Arbeitsmarkt vorzunehmen, sondern der Verweis auf andere europäische Länder mit einem vergleichbaren sozialen Sicherungsniveau reicht aus, um die Richtung für einen erfolgreichen Beschäftigungsaufbau anzuzeigen. Generell gilt, dass Länder mit einer hohen Erwerbsbeteiligung der Frauen, die zumeist im Dienstleistungssektor tätig sind, gleichzeitig gute Arbeitsmarktdaten vorweisen.

Während die meisten westeuropäischen Länder in den letzten Jahren beschäftigungspolitische Fortschritte gemacht haben, die zu einem Abbau der Arbeitslosigkeit geführt haben (in Ländern wie der Schweiz, den Niederlanden oder Norwegen herrscht inzwischen Vollbeschäftigung), verharrt Deutschland in internationalen Beschäftigungsrankings auf den letzten Plätzen (vgl. Eichhorst et al. 2001, Bertelsmann Stiftung 2002). Besonders negativ ragt das hohe Ausmaß der Langzeitarbeitslosigkeit (vor allem bei gering qualifizierten und älteren Arbeitskräften) heraus.

Möglichkeiten für eine neue Beschäftigungsdynamik in Deutschland bietet wie auch in den vergleichbaren Ländern allein der Dienstleistungssektor. Im weltmarktexponierten verarbeitenden Gewerbe gibt es in Deutschland, bezogen auf die Bevölkerung, mehr Arbeitsplätze als in vergleichbaren Gesellschaften; umso schwächer ist dafür die Beschäftigung im Dienstleistungsbereich entwickelt, insbesondere bei den einfachen, personenbezogenen und gering produktiven Dienstleistungen. Dies hat auch die „Hartz-Kommission“ jüngst festgestellt: „In Deutschland ist ein erhebliches Potential an Dienstleistungen aller Lohngruppen in verschiedenen Berufen unerschlossen. Private Dienstleistungen werden in größerem Umfang und legaler Form jedoch nur nachgefragt, wenn die Kosten in einem verträglichen Verhältnis zu dem verfügbaren Einkommen stehen. Dies ist heute oftmals nicht der Fall. Als Folge entstehen die Beschäftigungsverhältnisse in der Schattenwirtschaft“ (Kommmission 2002: 41). Es kann an dieser Stelle nicht diskutiert werden, ob die „Ich- und Familien-AG“ sowie die „Mini-Jobs“ attraktive Optionen für die legale Schaffung von Arbeitsplätzen im Dienstleistungssektor sind. Interessant ist aber, dass auch die „Hartz-Kommission“ für ein Recht auf „,einfache“ Arbeit plädiert und neue Wege in ein Beschäftigungsverhältnis fordert, dann allerdings vor einer strukturellen Öffnung des Arbeitsmarktes zurückschreckt, wohl um den Konsens der Akteure in Wahlkampfzeiten nicht zu gefährden.

Der Arbeitsmarkt- und Beschäftigungspolitik muss es vorrangig darum gehen, eine anderen Ländern vergleichbare Beschäftigungsdynamik im Dienstleistungssektor freizusetzen. 
Eine dynamische Expansion der Beschäftigung im Dienstleistungssektor könnte zu einem erheblichen Teil durch Überführung der (zunehmenden) Schwarzarbeit in die formelle Ökonomie gespeist werden. Sie könnte auch als Umwandlung der 325-Euro-Jobs in normale, nach betriebswirtschaftlichen statt nach sozialrechtlichen Gesichtspunkten organisierte Teil- und Vollzeitarbeit stattfinden. Durch Expansion der Beschäftigung im Dienstleistungssektor, und nur dadurch, ließen sich elementare Prinzipien der Beteiligungsgerechtigkeit realisieren, denen das derzeitige deutsche Beschäftigungssystem immer weniger gerecht wird. Zu diesen Prinzipien gehört zuallererst, dass jeder Bürger Zugang zu der privaten Autonomie und den sozialen Bindungen haben sollte, die in einer Gesellschaft wie der unseren über bezahlte Erwerbsarbeit vermittelt werden. „Bürgerarbeit“ ist ebenso wenig ein Ersatz für bezahlte Erwerbsarbeit wie Beschäftigung in einem künstlich am Leben gehaltenen ,Zweiten Arbeitsmarkt" oder gar eine noch so hohe Arbeitslosenunterstützung. Hohe Arbeitslosigkeit verletzt das Prinzip der Beteiligungsgerechtigkeit ebenso wie eine - unter anderem durch Verweigerung einer entsprechenden Betreuungsinfrastruktur (Ganztagsschulen!) - niedrig gehaltene Erwerbsquote von Frauen oder das Herausnehmen von Menschen aus dem Arbeitsmarkt durch Frühverrentung bzw. durch aus arbeitsmarktpolitischen Erwägungen künstlich verlängerte Ausbildungszeiten. Eine niedrige allgemeine Erwerbsquote - und insbesondere ein niedriges Beschäftigungsniveau im Bereich der personenbezogenen Dienstleistungen - führt zum Ausschluss gering qualifizierter Arbeitnehmer nicht nur aus Beschäftigung, sondern tendenziell aus der Gesellschaft insgesamt.

Die entscheidende Barriere gegen eine Expansion der Dienstleistungsbeschäftigung ist die so genannte Kostenkrankheit des Dienstleistungssektors. Diese geht darauf zurück, dass infolge des unvermeidlich geringeren Produktivitätswachstums im Dienstleistungssektor im Vergleich zur Industrie bei annähernd gleichem Wachstum der Löhne die realen Preise von - notwendig personalintensiven - Dienstleistungen schneller steigen müssen als die von immer kapitalintensiver produzierten Industriegütern. Zugleich gilt, dass die Nachfrage gerade nach personen- und haushaltsbezogenen Dienstleistungen in hohem Maße preisabhängig ist: Sind die Preise zu hoch oder steigen sie, so werden Dienstleistungen, die ansonsten am Markt gehandelt werden könnten, entweder nicht mehr nachgefragt, im Haushalt in Eigenleistung erstellt, in Schwarzarbeit produziert oder, in Deutschland, in die graue 325Euro-Zone abgedrängt. Der in Deutschland dynamisch wachsende Sektor der Schattenwirtschaft hat die gigantische Größe von „bis zu 300 Mrd. Euro erreicht. Dieses Volumen ist der Steuer- und Sozialversicherungslast entzogen. In Deutschland existieren rund 5 Mill. Vollzeitstellen in der Illegalität. Im Bereich der privaten Haushaltshilfen stehen rund 40000 gemeldeten ca. 3,3 Mill. tatsächliche Beschäftigungsverhältnisse gegenüber" (Kommission 2002: 40; vgl. auch F. Schneider in diesem Heft).

Das Argument der Kostenkrankheit gilt weniger für hoch qualifizierte produktionsnahe und zunehmend exportierbare Dienstleistungen; in diesem Bereich ist das Beschäftigungsniveau in Deutschland auch vergleichsweise hoch und wird darüber hinaus wahrscheinlich statistisch unterschätzt. Die insgesamt dennoch niedrige deutsche Erwerbsquote lässt dann jedoch eine Auseinandersetzung mit den wirtschaftlichen Voraussetzungen von Beschäftigungswachstum bei einfachen, personenbezogenen und gering produktiven Dienstleistungen umso dringlicher erscheinen. Letztere sind im Übrigen genau die Tätigkeiten, in denen gering qualifizierte Arbeitnehmer angesichts des von der Industrie irreversibel eingeschlagenen Rationalisierungspfades „,nach oben“ noch am ehesten Arbeit finden könnten. Da in der öffentlichen Diskussion der so genannte „Niedriglohnsektor“ ebenfalls hier lokalisiert wird, beziehen sich die vorliegenden Thesen ausschließlich auf diesen Bereich. 


\section{Strategische Optionen}

Will man den Dienstleistungssektor als Vehikel für eine Politik der Beschäftigungsexpansion nutzen, so muss man die relativen Preise von - beschäftigungsintensiven - Dienstleistungen senken. Dies geht grundsätzlich auf zweierlei Weise: durch Senkung der auf Dienstleistungen erhobenen Verbrauchssteuern oder der bei der Produktion von Dienstleistungen anfallenden Arbeitskosten. Letzteres wiederum kann sowohl durch Senkung der Bruttolöhne der im Dienstleistungssektor Beschäftigten geschehen, was zu einer größeren gesamtwirtschaftlichen oder intersektoralen Lohnspreizung führt, oder durch Senkung der Sozialversicherungsbeiträge auf niedrige Einkommen bzw. auf die Einkommen von Arbeitnehmern in ausgewählten beschäftigungsintensiven Bereichen des Dienstleistungssektors, mit der Folge einer größeren Spreizung, nicht der Löhne, sondern der Arbeitskosten.

Eine Expansion des öffentlichen Sektors und der öffentlichen Beschäftigung, einschließlich des so genannten ,Zweiten Arbeitsmarkts“, bietet als solche keinen Ausweg. Auch die öffentlichen Dienstleistungen leiden unter der Kostenkrankheit des Dienstleistungssektors; dies ist der Grund für den heute in allen europäischen Ländern wirksamen Zwang zur Haushaltskonsolidierung und zur Privatisierung weiter Bereiche der Infrastruktur. Auch deshalb gibt es, wenn das Ziel darin besteht, das Niveau der Beschäftigung zu erhöhen, zu einer Politik der Förderung des privaten Dienstleistungssektors keine Alternative. Gerade in Deutschland wird es wegen der Staatsverschuldung sowie der Bindung der Fiskalpolitik an den europäischen Stabilitätspakt keine Möglichkeit geben, eine Lösung der Beschäftigungskrise durch Ausweitung der Staatstätigkeit und der öffentlichen Beschäftigung zu erreichen. Im Gegenteil hat in Deutschland die öffentliche Beschäftigung in den letzten Jahren abgenommen und wird mit Sicherheit weiter abnehmen. In einer Reihe von Gemeinden im Osten Deutschlands werden lebenswichtige Funktionen der kommunalen Versorgung nur noch durch Inanspruchnahme von - für die Kommunen - billigen ABM-Kräften wahrgenommen werden können, d.h. von Arbeitslosen, deren Leistung durch die Beiträge nicht arbeitsloser Arbeitnehmer an die Arbeitslosenversicherung finanziert wird. Diese Situation wird nicht dadurch weniger absurd, dass sie mittlerweile zur Normalität geworden ist.

Ein Versuch, die relativen Preise arbeitsintensiver Dienstleistungen durch eine selektive Senkung der Verbrauchssteuern zu senken, ist neben anderen europäischen Staaten (Europäische Kommission 1999) Ende der 90er Jahre auch von der französischen Regierung unternommen worden. Dabei wurde der Mehrwertsteuersatz für die Instandhaltung von Gebäuden und Wohnungen von 20,5 auf 5,5 \% reduziert. Eine Erhöhung der Nachfrage nach Arbeit, die in diesem Sektor naturgemäß zu einem großen Teil gering qualifizierte Arbeit sein wird, kann allerdings nur dann eintreten, wenn die Steuersenkung an die Kunden weitergegeben und als Folge die abgesetzte Menge an Dienstleistungen vergrößert wird; dies setzt ein Mindestmaß an Wettbewerb und freiem Marktzugang voraus. In Deutschland wäre deshalb als flankierende Maßnahme an eine Lockerung der Handwerksordnung zu denken, wie es etwa die Monopolkommission vorgeschlagen hat.

Die folgenden Ausführungen konzentrieren sich auf die beiden Möglichkeiten zur Senkung der Arbeitskosten, die entweder als Alternative oder als Ergänzung zu Steuersenkungen realisiert werden könnten. Begonnen wird mit der Einrichtung eines so genannten Niedriglohnsektors. Anschließend werden die Möglichkeit diskutiert, die Beschäftigung niedrig produktiver Arbeitnehmer durch eine Reform der Sozialabgaben zu verbessern. 


\section{Senkung der Bruttolöhne von Arbeitnehmern mit niedriger Produktivität}

Eine Reihe von Arbeitsmarktexperten neoklassischer Ausprägung vertritt die Auffassung, dass eine Senkung der Arbeitskosten durch Senkung der Bruttolöhne, und nur diese, die Beschäftigung durch Schaffung eines Niedriglohnsektors erhöhen würde. In Deutschland würde jede Senkung des Bruttolohnniveaus, wenn der durch sie zu schaffenden Nachfrage nach Arbeit ein entsprechendes Angebot gegenüberstehen soll, eine gleichzeitige Senkung der Regelsätze der Sozialhilfe erfordern. Ergebnis wäre, dass eine erhebliche Anzahl von Arbeitnehmern Arbeitseinkommen erzielen würden, die unterhalb eines des sozialen Mindestniveaus lägen. Soweit dies als unerwünscht angesehen würde, müssten sozialpolitische Instrumente entwickelt werden, mit denen Bezieher von Armutseinkommen auf das Mindestniveau der Lebenshaltung, das die Gesellschaft allen ihren Mitgliedern zu garantieren bereit ist, hinaufgeschleust werden. Einrichtung oder Duldung eines Niedriglohnsektors geht deshalb typischerweise mit Programmen nach Art des amerikanischen „Earned Income Tax Credit“" zusammen. Derartige Sozialleistungen werden in der Regel von Einkommenssteigerungen bis zu einer gewissen Obergrenze nicht ganz aufgezehrt und ihren Empfängern nur schrittweise entzogen (,,progressiver Leistungsentzug“). Dies macht sie bei Ökonomen zu Recht populär. Es ändert aber nichts daran, dass sie eine Absenkung regulärer Arbeitseinkommen in die Armutszone voraussetzen.

Die politische Förderung eines Niedriglohnsektors verstößt gegen das Prinzip der Lohnoder Leistungsgerechtigkeit, das noch am ehesten von allen Parteien, unabhängig von den jeweiligen Interessen, gemeinsam so lange geduldet werden könnte, wie folgende Regel gilt: dass nämlich niemand, der im Rahmen des Zumutbaren unter Einsatz aller ihm zur Verfügung stehenden Kräfte Arbeit leistet, am Ende seines Arbeitstages weniger verdient haben sollte, als er benötigt, um am Leben seiner Gesellschaft menschenwürdig teilnehmen zu können. Wer tut, was ihm möglich ist, sollte, in anderen Worten, nicht arm sein also z. B. nicht beim Sozialamt einen Antrag auf eine Sonderzuwendung stellen müssen, wenn die Kinder zu Weihnachten neue Sportschuhe bekommen sollen. Die gute Nachricht ist, dass eine solche Verletzung elementarer Prinzipien von Leistungsgerechtigkeit zur Behebung der Beschäftigungskrise in unserer Gesellschaft nicht nötig ist.

\section{Senkung der Sozialversicherungsbeiträge von Arbeitnehmern mit niedriger Produktivität}

Als Alternative zu einer Senkung der Bruttolöhne steht - vor allem in Ländern wie Deutschland, in denen die staatlich verordneten Lohnnebenkosten besonders hoch sind - eine Senkung der Sozialversicherungsbeiträge auf niedrige Einkommen zur Verfügung. Die dadurch zu erreichende selektive Senkung der Lohnnebenkosten kann die Kosten einfacher Arbeit stark genug vermindern, um die Kosten der Produktion von Dienstleistungen und damit, bei Wettbewerb, die relativen Preise derselben beschäftigungsfördernd zu senken, ohne dass zugleich eine Senkung der Bruttolöhne erforderlich wäre. Reformen des Sozialstaats, insbesondere der Art und Weise seiner Finanzierung, können insofern zur Verbesserung der Beschäftigungsfähigkeit der Volkswirtschaft beitragen.

Ein hoher vom Staat zur Finanzierung sozialer Solidarität in Anspruch genommener Anteil an Einkommen, die auf niedrigem Produktivitätsniveau erwirtschaftet werden, erhöht die Produktivitätsschwelle, die ein Arbeitnehmer erreichen muss, um in bezahlte Beschäfti- 
gung eintreten zu können. Wer in Deutschland 1000 Euro brutto verdient, behält nach Abzug seiner Sozialversicherungsbeiträge etwa 800 Euro netto übrig; sein Arbeitgeber muss für ihn insgesamt, unter Einrechnung der Arbeitgeberbeiträge, 1200 Euro aufwenden. Wer für einen Nettolohn von 800 Euro einen Betrag von 1200 Euro erwirtschaften muss, zahlt damit de facto eine „Beschäftigungssteuer“ von $33 \%$ auf seinen Gesamtlohn, die ohne jede Progression auf alle Einkommen jenseits von 325 Euro erhoben wird. Geht man davon aus, dass bei einem Sozialhilfesatz von etwa 600 Euro die Aufnahme einer Vollzeittätigkeit unterhalb eines zu erwartenden Nettoeinkommens von 800 Euro nicht lohnt, so führen Höhe und Struktur der gegenwärtigen Belastung niedriger Einkommen durch Sozialabgaben dazu, dass Personen, deren Produktivität nicht ausreicht, ein Produkt in Höhe von mindestens 1200 Euro zu erwirtschaften, von bezahlter Beschäftigung ausgeschlossen bleiben, und zwar obwohl ihre mögliche Arbeitsleistung als solche ihnen ein selbst verdientes Einkommen weit oberhalb der durch die Sozialhilfe definierten Armutsgrenze - in dem Bereich von 800 bis 1200 Euro - verschaffen könnte.

Um eine derartige faktische Ausgliederung aus dem Arbeitsmarkt und die damit verbundene dauerhafte Marginalisierung wenigstens zum Teil aufzuheben, hat die Hartz-Kommission so genannte „Mini-Jobs“ vorgeschlagen und damit die zu Beginn der rot-grünen Regierungspolitik formulierte Neufassung der damaligen 630-DM-Regelung wieder deutlich korrigiert. Wohl auch weil es nicht gelungen ist, die 325-Euro-Jobs ,auszuhungern“, wie es große Teile der Gewerkschaften und der SPD vorhatten, soll nun die Geringfügigkeitsgrenze für Beschäftigungen in privaten Haushalten auf 500 Euro angehoben, der Beitragseinzug für geringfügige Beschäftigungen vereinfacht und auch die steuerliche Förderung von Dienstleistungen in privaten Haushalten eingeführt werden (vgl. Bundestag-Drucksache 14/9946). In dieser Frage scheinen sich also einige der politischen Akteure bewegt zu haben, nachdem noch 1999/2000 im Bündnis für Arbeit die von der Benchmarking-Gruppe vorgelegten Empfehlungen zur Verbesserung der Beschäftigungschancen von gering qualifizierten Arbeitnehmern (vgl. Fels et al. 1999, Streeck und Heinze 1999) eine Blockadehaltung vor allem bei den Gewerkschaften ausgelöst hatte. Ein wesentlicher Grund für das Aufbrechen (wenigstens auf Kommissionsebene) des klassischen defensiven Instrumentariums zum Ausgleich von Angebot und Nachfrage am Arbeitsmarkt dürfte in der äußerst mageren Bilanz liegen, die die rot-grüne Regierungspolitik vorweisen kann. Sowohl die über 4 Mill. registrierten Arbeitslosen im Juli und August 2002 (der höchsten Zahl in der Amtszeit Schröders) als auch die anhaltenden Meldungen über Insolvenzen und massenhaften Stellenabbau in nahezu allen Branchen haben den Vorschlägen der Kommission „Moderne Dienstleistungen am Arbeitsmarkt" aus Sicht der Bundesregierung eine neue strategische Rolle zugewiesen. Ob die Ergebnisse aber in reale Politik umgemünzt werden oder nur symbolische Politik bleiben, wird erst nach den Bundestagswahlen abzusehen sein.

Ein System der sozialen Sicherung, das zwar dem Versicherungsprinzip folgt, jedoch sozial ausgrenzende Wirkungen hat, verstößt gegen ein weiteres moralisches Prinzip, das der $B e$ lastungsgerechtigkeit. Dieses besagt, dass die Gemeinschaft keines ihrer Mitglieder mit Solidaritätspflichten belasten darf, die so hoch sind, dass es unter ihnen zusammenbricht d.h. die Fähigkeit verliert, für sich selber zu sorgen. Ein Sozialstaat, der das für formale Beschäftigung mindestens erforderliche Produktivitätsniveau so stark heraufsetzt, dass weniger leistungsfähige Personen keine Beschäftigung finden können oder gezwungen sind, ihren Lebensunterhalt, wenn sie sich nicht von der Gesellschaft alimentieren lassen wollen, durch Schwarzarbeit oder 325-Euro-Arbeit zu verdienen - ein Sozialstaat also, dessen 
institutionelle Ordnung am Arbeitsmarkt gegen gering qualifizierte Arbeitnehmer und Bezieher niedriger Einkommen diskriminiert - bedarf nicht nur aus wirtschaftlichen, sondern auch und vor allem aus moralischen Gründen dringend der Reform.

Im Übrigen gilt: Wenn in Deutschland, wie in zahlreichen anderen Ländern, die soziale Sicherung der Arbeitnehmer ganz oder zum überwiegenden Teil durch Steuern statt durch Beiträge finanziert würde, verstünde sich die Beachtung des Prinzips der Belastungsgerechtigkeit von selbst. Bei einer Finanzierung der sozialen Sicherung durch die Einkommensteuer kämen den Beziehern niedriger Einkommen auch in Bezug auf ihren Solidaritätsbeitrag zur sozialen Sicherung die entlastenden Wirkungen eines Freibetrags und einer Progressionszone zugute; dies ist seit Anfang der 90er Jahre z. B. in den Niederlanden weitgehend der Fall. In ausgebauten Sozialstaaten gibt es als Alternative zu einer das Prinzip der Leistungsgerechtigkeit verletzenden Förderung eines Niedriglohnsektors die Möglichkeit einer Behandlung von Sozialabgaben als Steuern und damit einer selektiven Entlastung niedriger Einkommen von Sozialabgaben bei Gewährung gleicher und uneingeschränkter (,Bürger“-)Rechte auf soziale Sicherung und Solidarität.

Die Einrichtung eines Freibetrags und einer Progressionszone bei den Sozialversicherungsbeiträgen wird zu Unrecht, und in leicht durchschaubarer polemischer Absicht, als Subventionierung bezeichnet. Eine Entlastung von Abgaben, die das Prinzip der Belastungsgerechtigkeit verletzen, indem sie Bürgern die Möglichkeit nehmen, mit ihrer Arbeit für sich selbst zu sorgen, ist ebenso wenig eine Subventionierung wie die Einrichtung eines Freibetrags bei der Einkommensteuer. Niemand kommt auf die Idee, von Subventionierung zu sprechen, weil die Kinder gering verdienender Arbeitnehmer auch dann öffentliche Schulen besuchen können, wenn das Einkommen der Familie unterhalb der Freibetragsgrenze des Steuerrechts liegt und die Familie deshalb keine Einkommensteuer zahlt. Ähnlich kann und muss die überfällige Beseitigung der dreiunddreißigprozentigen Beschäftigungssteuer für Geringverdienende als Erfüllung eines Anspruchs auf gerechte Lastenverteilung verstanden und darf nicht, wie dies gerade von Gewerkschaftsseite ständig geschieht, semantisch als wirtschaftlich ineffiziente Verteilung von staatlichen Wohltaten an Klientengruppen denunziert werden.

Von Mitgliedern der Arbeitsgruppe Benchmarking und anderen ist vorgeschlagen worden, die ohnehin geplante teilweise Umstellung der Finanzierung der sozialen Sicherung von Beiträgen auf Steuern, deren Ziel eine Senkung der Lohnnebenkosten und die von ihr erwartete anschließende Erhöhung des Beschäftigungsniveaus ist und deren Notwendigkeit von niemandem bestritten wird, statt zu einer linearen zu einer selektiven Senkung der Sozialversicherungsbeiträge zu nutzen. Mit dem Aufkommen der Ökosteuer, die zu einer allgemeinen Senkung der Sozialversicherungsbeiträge genutzt wurde, wäre es bei entsprechender Ausgestaltung möglich gewesen, alle Arbeitnehmereinkommen bis rund 750 Euro pro Monat von Sozialversicherungsbeiträgen freizustellen und eine Progressionszone einzurichten, in der der Beitrag allmählich auf das Normalniveau gestiegen wäre. Maßnahmen dieser Art wären selbstverständlich auch bei den geplanten weiteren Schritten zur Beitragsentlastung mit Hilfe steigender Energiesteuern möglich gewesen; die Grenzwerte für Freibetrag und Progressionszone lassen sich je nach Umfang der zur Verfügung stehenden Mittel festsetzen (vgl. Fels et al. 1999: 41 ff., Eichhorst et al. 2001). Auf jeden Fall haben die von 1999 bis Ende 2002 praktizierten und umgesetzten linearen Senkungen der Beiträge nicht zu zusätzlicher Beschäftigung geführt. 
Eine selektive Senkung der Sozialversicherungsbeiträge auf niedrige Einkommen würde zu einer wünschenswerten Spreizung der Arbeitskosten ohne gleichzeitige Spreizung der Nettoeinkommen führen. Im Gegenteil würden die Nettolöhne von Geringverdienern wegen des Wegfalls der vom Arbeitnehmer zu entrichtenden Sozialversicherungsbeiträge wahrscheinlich zunehmen, auch wenn die Entlastung teilweise an die Nachfrageseite durchgereicht würde. Ebenso wie bei einer Senkung der Einkommensteuer auf niedrige Einkommen oder bei einer Senkung der Unternehmenssteuern würde es sich bei einer am unteren Ende des Einkommensspektrums konzentrierten selektiven Entlastung von Sozialversicherungsbeiträgen nicht um ein Förderprogramm für „Problemgruppen“ handeln, bei dem es um ,Zielgenauigkeit“" und um die Verhinderung von „Mitnahmeeffekten“ zu gehen hätte; derartige Forderungen sind zu Recht weder bei der Einkommensteuer- noch bei der Unternehmenssteuerreform vorgetragen worden. Vielmehr ginge es um eine langfristige Veränderung der Rahmenbedingungen für die Nachfrage nach einfacher Arbeit bzw. den Zugang von gering qualifizierten Arbeitnehmern zu bezahlter Beschäftigung im ersten Arbeitsmarkt. Mit etwas Phantasie würde es auch gelingen, grobe „Mitnahmen“ innerhalb gut verdienender Haushalte zu vermeiden bzw. zu begrenzen (vgl. Fels et al. 1999: 68). Zusammengefasst würde es sich um eine Maßnahme der Wirtschafts- und nicht der Sozialpolitik handeln. Hier liegt auch die Abgrenzung zu dem so genannten „Mainzer Modell“, bei dem Niedriglohnempfänger degressiv gestaffelte Zuschüsse erhalten. Auch wenn das Programm inzwischen auf alle Bundesländer ausgedehnt wurde, bleibt es ein Problemgruppenprogramm, dessen Wirkungen äußerst bescheiden sind (im Sommer 2002 wurden nur etwas über 2150 Personen gefördert, während der Arbeitsminister noch immer von einem ,realistischen“ Potential von 30000 Förderfällen spricht).

Nach Berechnungen im Auftrag des IAB verhält sich der Beschäftigungseffekt einer nichtlinearen zu dem einer linearen Senkung von Sozialversicherungsbeiträgen wie etwa $3: 1$. Dies allein müsste Grund genug sein, die Senkung der Sozialversicherungsbeiträge asymmetrisch auszugestalten. Hinzu kommt, dass Berechnungen dieser Art schon deshalb extrem konservativ sind, weil sie den von einer nichtlinearen Beitragssenkung zu erwartenden Strukturbruch (Entstehung neuer Firmen, die neue Märkte erschließen, indem sie neue Produkte anbieten und für ihre Erstellung neue Prozesse anwenden) unberücksichtigt lassen müssen. Die in den letzten Monaten zwischen verschiedenen wirtschaftspolitischen Instituten, Interessengruppen und auch politischen Parteien eskalierten Streitigkeiten um den „Niedriglohn“ (und speziell die Berechnungen der angeblich enormen fiskalischen Kosten einer flächendeckenden Förderung von Arbeitnehmern und Arbeitgebern ohne Zielgruppenbindung) sind von ihren normativen Annahmen allesamt mehr als hinterfragbar. Sie sind ein weiterer Beleg dafür, wie die etablierten Akteure unseres hochsubventionierten und dennoch blockierten Arbeitsmarktes ihre Interessen verteidigen.

Durch eine Erhöhung gerade niedriger Einkommen und durch erhöhte Partizipation an bezahlter Erwerbsarbeit könnte im Übrigen ein Nachfrageeffekt ausgelöst werden, der weitere Beschäftigung schaffen könnte. Zunächst nicht begünstigt würden die besser verdienenden Arbeitnehmer, deren Sozialversicherungsbeiträge trotz steigender Belastung durch die Energiesteuer gleich bleiben würden. Auf längere Sicht freilich würde ihnen die verringerte Arbeitslosigkeit in Gestalt einer allgemeinen Senkung der Beiträge zur Arbeitslosenversicherung zugute kommen. Dasselbe gilt für die zu erwartenden Beitragseinnahmen aus zusätzlicher Beschäftigung sowie für die sinkenden Preise einfacher Dienstleistungen, die unter anderem eine Erhöhung des Arbeitsangebots von Frauen ermöglichen würden. 


\section{Schlussbemerkung}

Als Alternative zu einem „Niedriglohnsektor“ lassen sich eine breite Expansion der Beschäftigung und eine Steigerung der Partizipationsrate in der formalen Ökonomie durch eine Senkung der auf niedrige Löhne erhobenen Sozialabgaben erreichen. Diese wäre ein entscheidender Schritt auf dem Weg von einer Arbeitskraft stillegenden zu einer aktivierenden Arbeitsmarktpolitik, wie sie von der Hartz-Kommission gefordert wurde. Zielgruppenspezifische Programme und Sozialhilfe für die Schwächsten der Gesellschaft würden dabei nicht überflüssig. Aus- und Weiterbildungsmaßnahmen der aktiven Arbeitsmarktpolitik könnten jedoch viel mehr als bisher an bestehende Arbeitsverhältnisse und die von den Beschäftigten in ihnen gemachten Erfahrungen anknüpfen, was den besonderen Bedürfnissen gerade der Gruppe der Geringqualifizierten entgegenkommen würde. Und die Verfügbarkeit von offenen Stellen würde es Arbeitsamt und Sozialverwaltungen ermöglichen, ebenso wie in den sozialdemokratisch geprägten Ländern Skandinaviens die Bereitschaft zur Annahme zumutbarer Arbeit durch den Lackmustest eines realen Stellenangebots auf die Probe zu stellen. Eine die unteren Einkommen begünstigende redistributive Umgestaltung des Sozialstaats mit dem Ziel einer Erhöhung des Beschäftigungsniveaus im Rahmen einer aktivierenden Arbeitsmarktpolitik hat in zahlreichen europäischen Gesellschaften längst stattgefunden und steht insbesondere auch in den Ländern auf der Tagesordnung, wo wie in Deutschland niedrige Erwerbsquoten mit hoher Arbeitslosigkeit und hohen Lohnnebenkosten zusammenfallen. Maßnahmen dieser Art werden auch in den beschäftigungspolitischen Leitlinien der Europäischen Union vorgeschlagen, die Jahr für Jahr mit deutscher Zustimmung verabschiedet werden. Ähnlich hat sich der Internationale Währungsfonds bei seiner Begutachtung der wirtschaftlichen Lage und der Wirtschaftspolitik in Deutschland geäußert. Auch der Europäische Rat von Lissabon forderte im März 2000 eine deutliche Erhöhung der deutschen Beschäftigungsquote (Europäischer Rat 2000).

Von den deutschen Gewerkschaften, die damit in Europa allein stehen, wird eine selektive Senkung der Sozialversicherungsbeiträge auf niedrige Einkommen auf das Heftigste abgelehnt. Die Gründe hierfür sind nicht ganz klar. Ideologische Abwehrreflexe und manchmal offensichtlich gewollte Missverständnisse kommen zusammen mit einer bemerkenswerten Abwesenheit unabhängigen ökonomischen Sachverstands. Unter anderem führt dieses dazu, dass zur Bekämpfung einer niedrige Einkommen begünstigenden und in diesem Sinne progressiv umverteilenden Reform des Abgabensystems auf standardökonomische Argumente zurückgegriffen wird, die als alternative Lösung der Beschäftigungskrise gering qualifizierter Arbeitnehmer letztlich nur eine Senkung der Bruttolöhne offen lassen. Ebenfalls eine Rolle spielen dürften Sorgen um die Integrität des Äquivalenzprinzips bei der Sozialversicherung, an dem vor allem gut verdienenden Arbeitnehmern gelegen ist, sowie Misstrauen gegenüber jeder Maßnahme, die das Beitragsaufkommen der Rentenversicherung auch nur möglicherweise gefährden könnte. Hinzu kommt das kurzfristige Interesse der von den Gewerkschaften vor allem organisierten Industriearbeiterschaft an einer linearen Verteilung von Beitragssenkungen an alle Beitragszahler, nicht nur an Geringverdienende oder gar noch nicht Beschäftigte - ganz abgesehen von der Fixierung der Generation der über Fünfzigjährigen, die in den Gewerkschaften mangels Nachwuchses den Ton angeben, auf die Ziellinie des rechtsverbindlichen Rentenbescheids spätestens „mit sechzig“.

Eine die Bezieher niedriger Einkommen begünstigende und das Beschäftigungsniveau insbesondere zugunsten gering qualifizierter Arbeitnehmer und Einsteiger in die Erwerbsarbeit erhöhende Reform der Finanzierung der sozialen Sicherung dürfte deshalb in Deutschland 
auf absehbare Zeit keine Chance haben. Daran werden auch die Ergebnisse der Hartz-Kommission wahrscheinlich nicht viel ändern, die zwar unter günstigen politischen Rahmenbedingungen neue Akzente in der Arbeitsmarktpolitik in Richtung einer Aktivierung der Arbeitsförderung umsetzen könnten; eine wirkliche Reform stellen sie aber aufgrund der Begrenztheit und des Kompromisscharakters der Vorschläge nicht dar. Vor größeren institutionellen Reformen schreckt anscheinend aber nicht nur die offizielle Politik zurück; auch die gesellschaftlichen Großorganisationen wollen auf dem Arbeitsmarkt und generell im Bereich der sozialen Sicherungssysteme eher nur die Risiken begrenzen. Da den deutschen Wählern in den letzten Jahrzehnten eingeredet wurde, alles sei in Sicherheit und die Herausforderungen seien innerhalb der institutionellen Ordnung der Nachkriegsgesellschaft lösbar, ist es auch nicht überraschend, wie wenig die erforderlichen Dimensionen einer Umgestaltung des Arbeitsmarktes und der sozialen Sicherungssysteme im Alltag präsent sind (vgl. die Beiträge in Mayer 2002, Offe 2002). Die erforderlichen Veränderungen werden deshalb nur mühsam umgesetzt werden können und von vielen Konflikten und Krisen begleitet sein; der von der Hartz-Kommission eingeschlagene Weg stellt nur die erste Etappe auf dem langen Weg zu einer institutionellen Reform der Arbeitsmarktpolitik in Deutschland dar.

\section{Literaturverzeichnis}

Bertelsmann Stiftung (Hrsg.) (2002): Internationales Beschäftigungs-Ranking 2002. Gütersloh.

Bundestag-Drucksache 14/9946: Antrag: Neue Beschäftigung - schnelle Vermittlung erstklassiger Service. Reformvorschläge der Hartz-Kommission unverzüglich umsetzen. 11. September 2002. Berlin.

Eichhorst, W. et al. (2001): Benchmarking Deutschland: Arbeitsmarkt und Beschäftigung. Bericht der Arbeitsgruppe Benchmarking und der Bertelsmann Stiftung. Berlin, Heidelberg.

Europäische Kommission (1999): Commission Proposes Tax Cuts to Boost Jobs. Pressemitteilung IP/99/1002 vom 20.12.1999. Download unter: http://jpn.cec.eu.int/english/ info-resources/6-6-15-09.htm

Europäischer Rat (2000): Schlussfolgerungen des Gipfeltreffens „Beschäftigung. Wirtschaftsreformen und sozialer Zusammenhalt - hin zu einem Europa der Innovation und des Wissens“. Lissabon, 23. und 24. März 2000. Download unter: http://www.europarl. eu.int/summits/lis1 de.htm

Fels, G., R. G. Heinze, H. Pfarr und W. Streeck (1999): Möglichkeiten zur Verbesserung der Beschäftigungschancen gering qualifizierter Arbeitnehmer. Berlin. Download unter: http: //www.buendnis.de

Hartz-Kommission (2002): Moderne Dienstleistungen am Arbeitsmarkt. Mimeo. Berlin.

Jahoda, Marie, Paul F. Lazarsfeld und Hans Zeisel (1975): Die Arbeitslosen von Marienthal. Suhrkamp. Frankfurt a. M.

Mayer, K. U. (Hrsg.) (2002): Die Beste aller Welten? Marktliberalismus versus Wohlfahrtsstaat. Frankfurt a. M.

Offe, C. (2002): Unsicherheiten und Rückversicherungen. Die Mitbestimmung, 9, $40 \mathrm{ff}$.

Schmidt, H. (2002): Unkraut jäten und ackern. Die Zeit, 29. August 2002, $10 \mathrm{f}$.

Schneider, F. (2003): Zunehmende Schattenwirtschaft in Deutschland: Eine wirtschaftsund staatspolitische Herausforderung. In diesem Heft, 148-159.

Streeck, W. und R. G. Heinze (1999): Runderneuerung des deutschen Modells. Aufbruch für mehr Jobs. In: H.-J. Arlt und S. Nehls (Hrsg.): Bündnis für Arbeit. Opladen, 147 ff. 